\title{
Anesthesia and postoperative delirium: the agent is a strawman - the problem is $\mathrm{CO}_{2}$
}

\author{
W. Alan C. Mutch, MD, FRCPC • Renée El-Gabalawy, MA, PhD
}

Received: 26 December 2016/Revised: 25 January 2017/ Accepted: 27 February 2017/Published online: 10 March 2017

(c) Canadian Anesthesiologists' Society 2017

\section{To the Editor,}

Postoperative delirium and cognitive dysfunction (POCD) are complex perioperative problems associated with substantial personal and societal costs. ${ }^{1}$ Proposed mechanisms of the neurotoxicity of anesthetic agents on the vulnerable brain have included their effects on $\mathrm{N}$ methyl-D-aspartate, glutamate, and $\gamma$-aminobutyric acid receptors, caspases, apoptosis, synaptogenesis, and neuronal circuit formation, among others. ${ }^{2}$ Early bench or small-animal research into anesthetic agents and neurotoxicity was associated with significant limitations, including failure of these models to necessarily translate to humans. Recent clinical studies and meta-analyses from several sources and varied approaches lead one to reconsider the linkages between anesthetic agents, neurotoxicity, and clinical outcome. ${ }^{3,4}$ Despite the absence of compelling support, large-scale studies that focus on anesthetic agents continue to be implemented, with few studies examining alternative explanations for these prevalent conditions.

W. A. C. Mutch, MD, FRCPC ( $\square)$

Department of Anesthesia and Perioperative Medicine, Kleysen Institute for Advanced Medicine Researcher, Health Sciences

Centre, University of Manitoba, Winnipeg, MB, Canada

e-mail: amutch@cc.umanitoba.ca

R. El-Gabalawy, MA, PhD

Departments of Clinical Health Psychology and Anesthesia \& Perioperative Medicine, Rady Faculty of Health Sciences, Max Rady College of Medicine, Winnipeg, MB, Canada

R. El-Gabalawy, MA, PhD

Departments of Psychology and Psychiatry, University of Manitoba, Winnipeg, MB, Canada
We propose that postoperative delirium and POCD are related, in part, to the conduct of the anesthetic, rather than to the anesthetic agents themselves. There is a lack of research evaluating the influence of changes in $\mathrm{CO}_{2}$ tension $\left(\Delta \mathrm{CO}_{2}\right)$ on the brain in regard to these conditions. With the advent of non-invasive, blood oxygenation leveldependent, magnetic resonance imaging (BOLD MRI) techniques, it has become clear that intracranial steal is common and is a potential risk factor for brain injury (see Figure). Major surgery is frequently associated with large swings in arterial $\mathrm{CO}_{2}$ tension. Cardiac, thoracic, major vascular, and major intra-abdominal surgery may be associated with a $\Delta \mathrm{CO}_{2}$ of $10-20 \mathrm{mmHg}$ or higher. The Figure shows areas of marked "steal" in the white matter unmasked by a $\Delta \mathrm{CO}_{2}$ of only $5 \mathrm{mmHg}$ in regions that correspond closely to those identified at autopsy with leukoaraiosis. This pathology is “... correlated with motor and gait disturbances, depressive symptoms, urinary disturbances, and some cognitive deficits." 5 We are currently conducting a feasibility study (NCT021226215) to assess linkages between intracranial steal as assessed by an MRI BOLD $\mathrm{CO}_{2}$ stress test and extensive preoperative neuropsychological testing to gain a thorough understanding of these processes.

If $\Delta \mathrm{CO}_{2}$-related changes in cerebral perfusion are found to be associated with cognitive deficits in ageing patients, could this mechanism be extended to another population at risk? Major surgery in children is also associated with large swings in $\mathrm{CO}_{2}$, and cerebrovascular reactivity (CVR) alterations are more pronounced in this age group. End-arteries are responsive in periventricular regions, related to regional hemorrhage, suggesting local vascular dysregulation. In addition, posterior fossa CVR is more robust, ${ }^{6}$ and cognitive links in neonates have been related to delayed cerebellar development. ${ }^{7}$ Research 


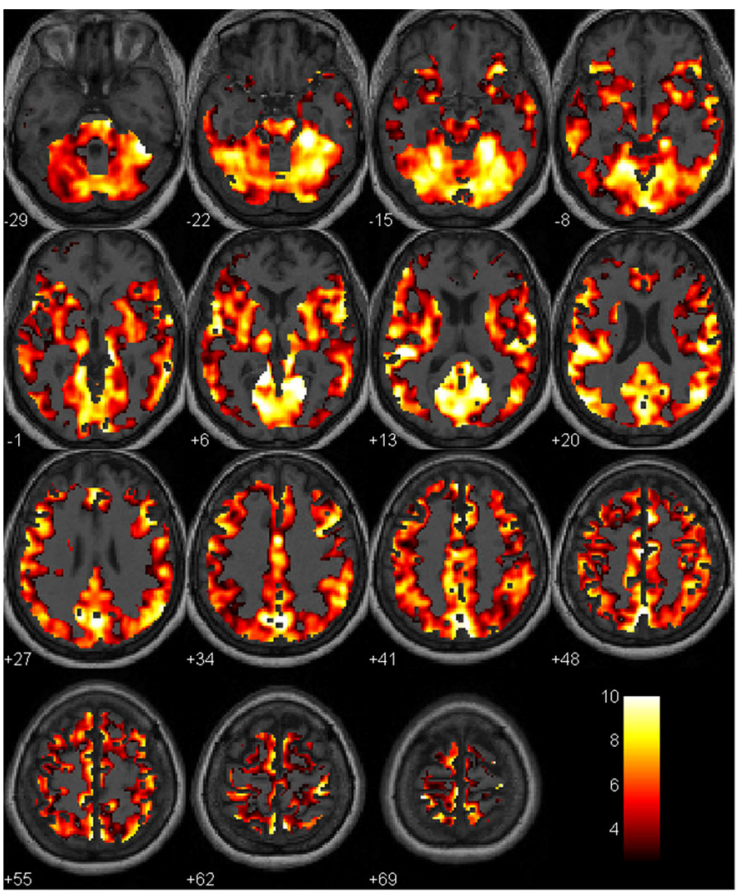

BOLD response to Hypercapnia

Figure Blood oxygenation level-dependent, magnetic resonance imaging (BOLD MRI) $\mathrm{CO}_{2}$ stress testing performed preoperatively in a patient with postoperative delirium. General linear modeling by statistical parametric mapping with a repeating, mild $(5 \mathrm{mmHg})$ hypercapnic stimulus. The left panel shows the anticipated BOLD signal response to hypercapnia (hot colours indicate where the voxel responds to $\mathrm{CO}_{2}$ at the $P=0.001$ level). The numbers at the side of each image indicate the distance above or below the anteroposterior

addressing this hypothesis would be timely in light of the Food and Drug Administration's recent warning ${ }^{8}$ to minimize exposure to anesthetic agents and sedatives in children under three years of age and women in the third trimester of their pregnancy.

A strawman can be defined as a misrepresented argument or logical fallacy that can be recognized as an illogical argument following scrutiny and careful dissection. Historical evidence has made it abundantly clear that anesthetic agents are neurotoxic in animal models. Currently, however, mounting evidence suggests that this argument is weak in the clinical realm. We offer an alternate thesis. We maintain that delirium or POCD may be reduced by applying general neuroanesthesia principles - most importantly, proper management of intra- and postoperative $\mathrm{CO}_{2}$ - to patients at risk. This hypothesis is testable. Such a small change in anesthetic management is essentially risk-free. Also, if found efficacious, it could significantly affect patients' health, particularly in those deemed at risk, such as older adults and children, thereby reducing healthcare spending.

Conflict of interest None declared.

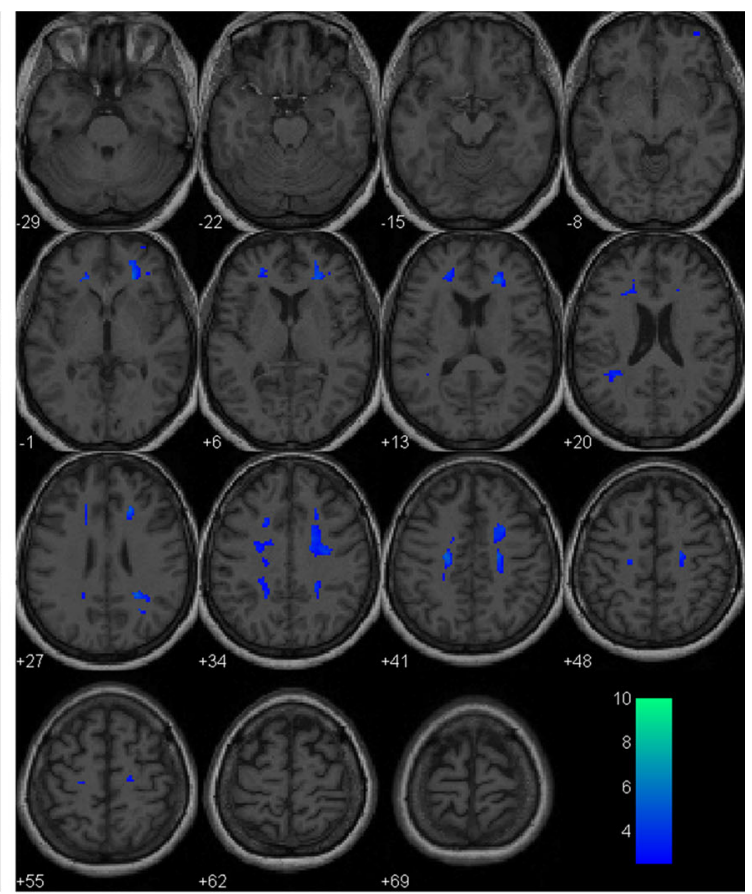

Intracranial Steal with Hypercapnia

commissure (in millimetres). The colour scale is the t-value score The right panel shows the inverse response in the same patient, highlighting regions of intracranial steal with the hypercapnic stimulus (a decrease in BOLD signal with hypercapnia and an increase with normocapnia). The areas at risk are predominantly in the white matter. These regions are thought to be at risk of perioperative ischemia with alterations in end-tidal $\mathrm{CO}_{2}$

Editorial responsibility This submission was handled by Dr. Gregory L. Bryson, Deputy Editor-in-Chief, Canadian Journal of Anesthesia

Funding Anesthesia Oversight Committee-Department of Anesthesia and Perioperative Medicine and University of Manitoba Start-up Funding.

\section{References}

1. Leslie DL, Marcantonio ER, Zhang Y, Leo-Summers L, Inouye SK (2008) One-year health care costs associated with delirium in the elderly population. Arch Intern Me 168: 27-32

2. Jevtovic-Todorovic $V$ (2016) General anesthetics and neurotoxicity: how much do we know? Anesthesiol Cli 34: 439-451

3. Graham MR, Brownell M, Chateau DG, Dragan RD, Burchill C, Fransoo RR (2016) Neurodevelopmental assessment in kindergarten in children exposed to general anesthesia before the age of 4 years: a retrospective matched cohort study. Anesthesiolog 125: 667-677

4. Mason SE, Noel-Storr A, Ritchie CW (2010) The impact of general and regional anesthesia on the incidence of post-operative cognitive dysfunction and post-operative delirium: a systematic review with meta-analysis. J Alzheimers Di 22(Suppl 3): 67-79

5. Pantoni L (2008) Leukoaraiosis: From an ancient term to an actual marker of poor prognosis. Strok 39: 1401-1403 
6. Ito H, Yokoyama I, Iida H, et al (2000) Regional differences in cerebral vascular response to $\mathrm{PaCO}_{2}$ changes in humans measured by positron emission tomography. J Cereb Blood Flow Meta 20: 1264-1270

7. Wagner M, Ryu YK, Smith SC, Patel P, Mintz CD (2014) Review: effects of anesthetics on brain circuit formation. J Neurosurg Anesthesio 26: 358-362
8. U.S. Food and Drug Administration. Drug Safety Communication: FDA review results in new warnings about using general anesthetics and sedation drugs in young children and pregnant women. Available from URL: https://www.fda.gov/downloads/ Drugs/DrugSafety/UCM533197.pdf (accessed February 2017). 